Department of Social Systems and Management

Discussion Paper Series

No.1288

Another View of Impact of BSE Crisis

in Japanese Meat Market through

the Almost Ideal Demand System Model

with Markov Switching

by

Satoshi KABE and Yuichiro KANAZAWA

February 2012

UNIVERSITY OF TSUKUBA

Tsukuba, Ibaraki 305-8573 JAPAN 


\title{
Another view of impact of BSE crisis in Japanese meat market through the almost ideal demand system model with Markov switching
}

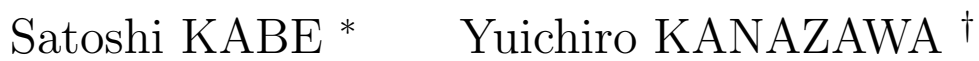

\begin{abstract}
During the period in this study, there have been serious food safety concerns regarding the Bovine Spongiform Encephalopathy (henceforth BSE) and bird flu in Japan. Previous studies such as Peterson and Chen (2005) and Ishida et al. (2006, 2010) assumed that structural change points in demands have already known and they specified transition functions at the change points. On the other hand, we detected the structural change points without any prior information about change points. To assess the structural change points in the Japanese meat market, we employed the Markov switching almost ideal demand system (MS-AIDS) model (Allais and Nichèle, 2007). In this study, we found the structural change point coinciding with the timing of first reported case of BSE, but not of bird flu.
\end{abstract}

${ }^{*}$ Doctoral Student, Graduate School of Systems and Information, University of Tsukuba. E-mail: k0420214@sk.tsukuba.ac.jp.

${ }^{\dagger}$ Professor of Statistics, Department of Social Systems and Management, University of Tsukuba. Email: kanazawa@sk.tsukuba.ac.jp. 


\section{Introduction}

Food safety issues (e.g., virus, bacteria, and toxic chemicals ) are said to influence the consumers' choices and preferences for food. Thus it is important to examine the change of consumption pattern as a results of reported food safety concerns.

In the Japanese meat market, there have been serious food safety concerns regarding to the Bovine Spongiform Encephalopathy (henceforth BSE) and bird flu. The BSE was initially recognized in cattle in the UK in 1986. The UK government announced that consumption of beef infected by BSE is suspected to cause new variant Creutzfeldt Jacob Disease (vCJD). Within the period from 1987 to 2002, the total number of infected cattle grew to 183,000 in the UK (Jin and Koo, 2003). The BSE crisis in the UK resulted in an immediate and significant decline in beef consumption throughout Europe including Ireland, Switzerland, France, Portugal, Belgium, and the Netherlands in the 1990s.

On September 2001, the Japanese government announced the first BSE case in the country. It was the first reported BSE case outside Europe. The cow believed to have the BSE was a five-year-old Holstein and was located in Chiba prefecture. The Japanese government expended more than one billion dollar over the subsequent half year to restore food safety (Peterson and Chen, 2005) and began BSE testing for all cattle in October 2001. It also banned imports of meat and bone meal to reduce the possibility of outbreak. However thirty six cases of BSE was confirmed during 2001 - 2009.

The bird flu is an infectious disease of birds (e.g., chicken, duck, and turkey) caused by avian influenza viruses. There are many subtypes of avian influenza viruses, but only some strains of four types have been highly pathogenic in human (e.g., H5N1 virus). The H5N1 virus is the most highly 
pathogenic strain. Many people infected with the H5N1 virus have died in Vietnam, Indonesia, Thailand and other Southeast Asia countries.

On January 2004, the first infection case with H5N1 virus in Japan was confirmed in Yamaguchi prefecture. Since then the chicken infected with H5N1 virus are periodically found in various regions in Japan. With the spread of bird flu in Japan, consumers are worried about the safety of chicken despite the fact that the Ministry of Health, Labor and Welfare in Japan announced that there is virtually no risk H5N1 infection from eating the infected chicken itself or the products made out of the infected chicken.

In the previous studies about structural change of demand in the Japanese meat market, for example, Jin and Koo (2003) identified a structural change point coinciding with the first BSE case in Japan by using the non-parametric tests. Peterson and Chen (2005) focused on the type and origin of beef products (i.e., wagyu, dairy, U.S., and Australian beef) and showed the difference of impacts on the beef products due to the first BSE case in Japan. Ishida et al. $(2006,2010)$ examined not only the structural change due to the BSE but also the bird flu in Japan and empirically investigated the difference of structural changes caused by BSE and bird flu.

Previous studies such as Peterson and Chen (2005) and Ishida et al. (2006, 2010) applied the gradual switching model (Ohtani and Katayama, 1986) to examine the nature of structural change in demands. This model utilizes a transition function to express a gradual shift pattern in demands under the assumption that starting-points of structural shift have already known. For example, the starting-points of structural shift in Ishida et al. (2006, 2010) were specified at the timing of first reported cases of BSE and bird flu in Japan. The authors assumed the four types of transition functions and selected a transition function which the likelihood is maximized. 
In this paper, we detect the structural change points without any prior information about change points in the market and at the same time examine when and how often the structure of demands changed. Also we compute the elasticities for price and expenditure to examine the change of consumers' behavior for meat products in Japan.

For these purposes, we employ the Markov switching almost ideal demand system (MS-AIDS) model proposed by Allais and Nichèle (2007). This model employs the Markov switching mechanism to capture the dynamic shift patterns. The Markov switching mechanism allows the frequent structural changes at random points, and it is suitable to uncover distinct dynamic patterns in different periods.

This paper is organized as follows. In section 2, we introduce the Markovswitching AIDS (MS-AIDS) model. Details of the model are in Appendix. In the next section, the estimation procedure is described. The data and results are presented in section 4 , and the last section contains the conclusion of this study.

\section{Markov-Switching AIDS Model}

Deaton and Muellbauer (1980) developed the almost ideal demand system (AIDS) model which is widely used in the empirical demand analysis even now, and several researchers extended the AIDS models (e.g., Cooper and McLaren, 1992; Banks et al., 1997; Moosa and Baxter, 2002; Ishida et al., 2006, 2010; Allais and Nichèle, 2007). The AIDS model is able to carry out the statistical test of theoretical constraints in demand theory, like the Rotterdam model (Theil, 1965) and applies the second-order Taylor expansion to the unknown functions, like the Translog model (Christensen et al., 1975). 
In the MS-AIDS model, representative consumer's demand for a category of products under consideration during the given time-period consists of a predetermined number of "regimes" or "states" and this model can estimate the degree of "belongingness" to these "regimes" by probability.

Suppose that $s_{t}$ is an unobserved random variable that takes an integer value in $1,2, \ldots, K$ to express "regime" or "state" at time $t$, then budget share of $i_{t h}$ product at time $t, \bar{w}_{i t}$ which is defined as $p_{i t} q_{i t} / m_{0 t}$ with price $p_{i t}$, quantity $q_{i t}$ and expenditure (or budget) $m_{0 t}\left(=\sum_{i} p_{i t} q_{i t}\right)$ takes the following form:

$$
\bar{w}_{i t}=\alpha_{i, s_{t}}+\sum_{j=1}^{N} \gamma_{i j, s_{t}} \log p_{j t}+\beta_{i, s_{t}} \log \left(\frac{m_{0 t}}{P_{t}}\right)
$$

where $P_{t}$ is a price index which is defined by

$$
\log P_{t}=\alpha_{0, s_{t}}+\sum_{k=1}^{N} \alpha_{k, s_{t}} \log p_{k t}+\frac{1}{2} \sum_{k=1}^{N} \sum_{j=1}^{N} \gamma_{k j, s_{t}} \log p_{k t} \log p_{j t}
$$

where $\alpha_{0, s_{t}}, \alpha_{i, s_{t}}, \gamma_{i j, s_{t}}$ and $\beta_{i, s_{t}}(i, j=1,2, \ldots, N)$ are regime-dependent parameters.

The parameters in (2.1) and (2.2) have the theoretical constraints ${ }^{1}$ as follows

$$
\begin{array}{cc}
\text { [Adding up] } & \sum_{i=1}^{N} \alpha_{i, s_{t}}=1, \sum_{i=1}^{N} \gamma_{i j, s_{t}}=0, \sum_{i=1}^{N} \beta_{i, s_{t}}=0, \\
\text { [Homogeneity] } & \sum_{j=1}^{N} \gamma_{i j, s_{t}}=0, \\
\text { [Symmetry } & \gamma_{i j, s_{t}}=\gamma_{j i, s_{t} .}
\end{array}
$$

1 "Adding up" guarantees that the total expenditure is equal to the sum of expenditures on the category of products under consideration. "Homogeneity" guarantees that if prices of products increase to $\tau p_{1 t}, \ldots, \tau p_{N t}$ for a scalar $\tau>0$, representative consumer has to increase his expenditure from $m_{0 t}$ to $\tau m_{0 t}$ to keep his utility level. "Symmetry" guarantees that the substitution effect in the Slutsky equation is symmetric. 
Following the previous studies (Rickertsen, 1996; Allais and Nichèle, 2007; Ishida et al., 2010), we include a trend effect, seasonal effect and habit effect into the intercept term $\alpha_{i, s_{t}}$ as

$$
\alpha_{i, s_{t}}=\bar{\alpha}_{i, s_{t}}+\nu_{i, s_{t}} t+\delta_{1, i} d_{1, t}+\delta_{2, i} d_{2, t}+\sum_{j=1}^{N} \phi_{i j} \bar{w}_{j, t-1}
$$

where $d_{1, t}$ and $d_{2, t}$ are dummy variables

$$
d_{1, t}=\left\{\begin{array}{ll}
1 & \text { if } t \text { is August } \\
0 & \text { otherwise }
\end{array} \quad d_{2, t}= \begin{cases}1 & \text { if } t \text { is December } \\
0 & \text { otherwise. }\end{cases}\right.
$$

As for seasonal effect, we set the dummy variables to adjust the seasonality in budget shares. The budget shares for meat and fish are considered to shift due to the seasonal habits (e.g., summer camp, gift-giving tradition, yearend party and so forth) in August and December. Furthermore, we include a habit effect which is defined as a linear function of one-lagged budget shares (Rickertsen, 1996; Allais and Nichèle, 2007). In order to satisfy the adding up condition, we impose the restriction $\sum_{i=1}^{N} \bar{\alpha}_{i, s_{t}}=1, \sum_{i=1}^{N} \nu_{i, s_{t}}=0$, $\sum_{i=1}^{N} \delta_{1, i}=\sum_{i=1}^{N} \delta_{2, i}=0$ and $\sum_{i=1}^{N} \phi_{i j}=0$. We also impose the restriction $\sum_{j=1}^{N} \phi_{i j}=0$ to avoid the identification problem.

The MS-AIDS model employs the Markov switching mechanism which is developed by Hamilton (1989). The Markov switching mechanism can express switching of regimes by using the unobserved random variables that follow the Markov process. To apply the Markov switching mechanism, we assume that transitions between regimes are governed by a K-state Markov chain with transition probabilities:

$$
\operatorname{Pr}\left(s_{t}=j \mid s_{t-1}=i\right)=\pi_{i j}, \quad i, j=1,2, \ldots, K
$$


and the transition matrix is defined as

$$
\boldsymbol{\Pi}=\left[\begin{array}{cccc}
\pi_{11} & \pi_{21} & \ldots & \pi_{K 1} \\
\pi_{12} & \pi_{22} & \ldots & \pi_{K 2} \\
\vdots & \vdots & \ddots & \vdots \\
\pi_{1 K} & \pi_{2 K} & \ldots & \pi_{K K}
\end{array}\right]
$$

where $\pi_{i 1}+\pi_{i 2}+\cdots+\pi_{i K}=1, i=1,2, \ldots, K$.

\section{Estimation}

The parameters of MS-AIDS model are estimated by iterating the following steps:

Step 0. Set the initial values of parameters and set $g=0$.

Step 1. Given the parameters at $g_{t h}$ iteration, calculate the conditional probabilities about the value of $s_{t}$ from the Hamilton filter (Hamilton, 1989).

Step 2. Calculate the score functions with respect to parameters ( see detail in Appendix ).

Step 3. Find the maximum likelihood estimates of parameters via $\mathrm{BHHH}$ algorithm (Berndt et al., 1974) and set $g=g+1$.

Step 4. Repeat steps 1 - 3 until the log-likelihood does not change.

In Allais and Nichèle (2007), authors estimate the transition probabilities by maximum likelihood estimation, but if transition probability has a boundary solution such as $\pi_{i j}=0$ or 1 , asymptotic normality of transition probabilities does not hold. To avoid this problem, we reparameterize the 
transition probability $\pi_{i j}, 0 \leq \pi_{i j} \leq 1$ as $\lambda_{i j}=\log \left(\pi_{i j} / \pi_{i K}\right)$, natural sufficient statistics for the multinomial model, $-\infty<\lambda_{i j}<\infty$ and calculate score function with respect to $\lambda_{i j}$. Once, we estimate the parameter $\lambda_{i j}$, then we calculate the transition probability $\pi_{i j}$ back using the standard formula

$$
\pi_{i K}=\frac{1}{1+\exp \left(\lambda_{i 1}\right)+\exp \left(\lambda_{i 2}\right)+\cdots+\exp \left(\lambda_{i K-1}\right)}
$$

and

$$
\pi_{i j}=\frac{\exp \left(\lambda_{i j}\right)}{1+\exp \left(\lambda_{i 1}\right)+\exp \left(\lambda_{i 2}\right)+\cdots+\exp \left(\lambda_{i K-1}\right)} .
$$

Assuming that budget share equations follow a multivariate normal distribution, Allais and Nichèle (2007) estimates variance-covariance matrix $\Sigma_{s_{t}}$ along with other parameters. However maximum log-likelihood becomes infinite if determinant of variance-covariance matrix $\left|\Sigma_{s_{t}}\right|$ goes to zero and numerical maximization algorithm (e.g., Newton-Raphson method) breaks down. Therefore we derive the maximum likelihood estimator $\widehat{\Sigma}_{s_{t}}$ after estimating all parameters other than $\boldsymbol{\Sigma}_{s_{t}}$ (see detail in Appendix).

\section{Data and Results}

The Ministry of Internal Affairs and Communications in Japan provides us with the household expenditure survey data (i.e., Family Income and Expenditure Survey). The household expenditure survey data includes the monthly time-series data about average expenditure and price of meat and fish products along with others. In this study, we used the average expenditure and price data of beef, pork, chicken and fish over January 1998 to December 2006 (108 months). Figure 1 plots the budget shares of meat products from January 1998 through December 2006. Fish, having consistently been the dominant source of protein for the Japanese for many years, has budget share 
of almost $50 \%$ of meat products (beef, pork, chicken, and fish). Therefore we omit the budget share of fish to reveal the budget share movement of meat products other than fish when we plot Figure 1.

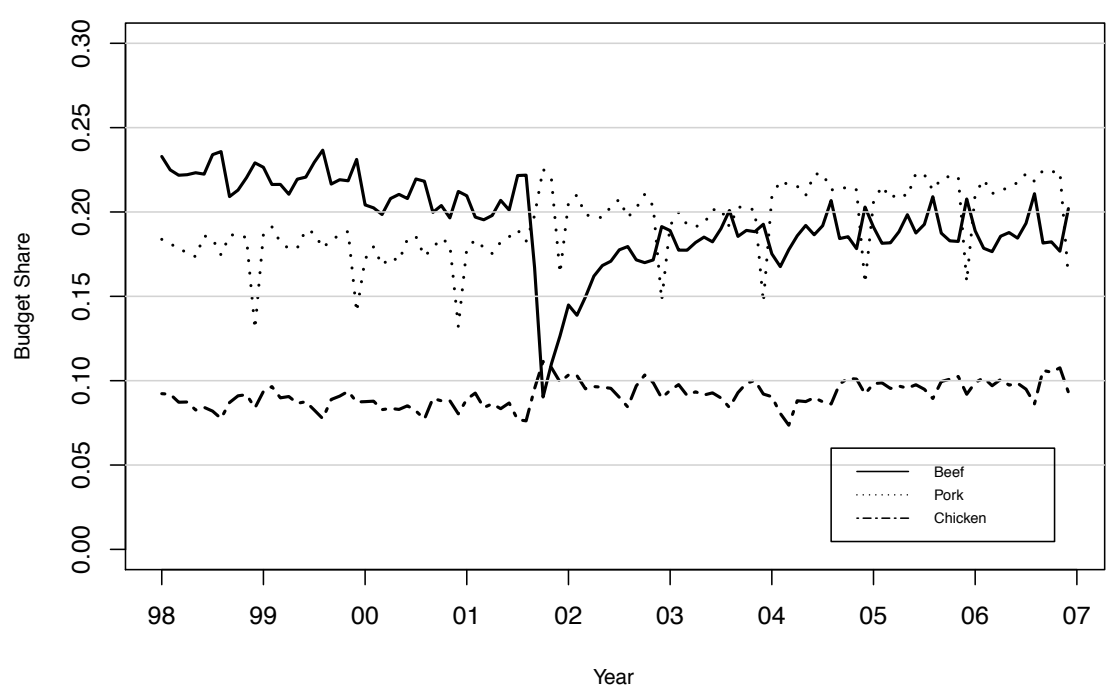

Figure 1: Plot of budget share data

During this period (see Figure 1), there exist the first reported case of BSE and of bird flu in Japan. The first BSE case was confirmed on September 2001 and beef consumption dwindled dramatically and then has gradually recovered. However the budget share of beef has not recovered at the same level as in the pre-BSE period. On the other hand, the change of budget share of chicken due to the first reported case of bird flu on January 2004 was relatively small compared with the change of beef demand due to the first BSE case. The budget share of chicken has recovered completely and even increased afterwards. Finally budget share of pork has gradually increased after the first BSE case on September 2001 but has experienced significant seasonal declines at the end of every year. 
Next we consider the seasonality in budget shares for meat products. To examine the seasonal variations in the budget shares, we calculate the average budget shares of meat products per month for January 1998 - December 2000 and for January 2003 - December 2006 respectively. This way we can avoid the influence of BSE. Figures 2 and 3 display the average budget shares of meat products per month for the two periods. During these periods, the average monthly budget shares for meat products do not significantly vary throughout the year except for the pork's decline in Decembers. Figures 2 and 3 also show the average monthly budget share for beef peaked in August.

In this study, therefore, we examine the following four models: model 1 only includes intercept parameter $\bar{\alpha}_{i, s_{t}}$. Model 2 includes seasonal effects on August and December into model 1, and model 3 adds a habit effect into model 2. Finally, model 4 further incorporates a trend effect into model 3.

Model $1 \alpha_{i, s_{t}}=\bar{\alpha}_{i, s_{t}}$

Model $2 \alpha_{i, s_{t}}=\bar{\alpha}_{i, s_{t}}+\delta_{1, i} d_{1, t}+\delta_{2, i} d_{2, t}$

Model $3 \alpha_{i, s_{t}}=\bar{\alpha}_{i, s_{t}}+\delta_{1, i} d_{1, t}+\delta_{2, i} d_{2, t}+\sum_{j=1}^{N} \phi_{i j} \bar{w}_{j, t-1}$

Model $4 \alpha_{i, s_{t}}=\bar{\alpha}_{i, s_{t}}+\nu_{i, s_{t}} t+\delta_{1, i} d_{1, t}+\delta_{2, i} d_{2, t}+\sum_{j=1}^{N} \phi_{i j} \bar{w}_{j, t-1}$

In Table 1, we computed the Akaike's information criterion (AIC) and likelihood ratio (LR) statistic ${ }^{2}$ for each of the candidate models when the number of regimes is two. The LR statistic shows that log-likelihood of model 4 is significantly higher than those of other candidate models, and also model 4 has a smallest AIC. Therefore we conclude that model 4 fits the data best.

\footnotetext{
${ }^{2} \mathrm{We}$ assumed the asymptotic normality of ML estimator (see detail in Bickel et al. (1998)) and applied the model selection criteria (e.g., AIC) and LR test. However, if nuisance parameters are not identified under the null hypothesis, LR test is not valid because the LR statistic does not have a standard asymptotic distribution (Garcia, 1998).
} 


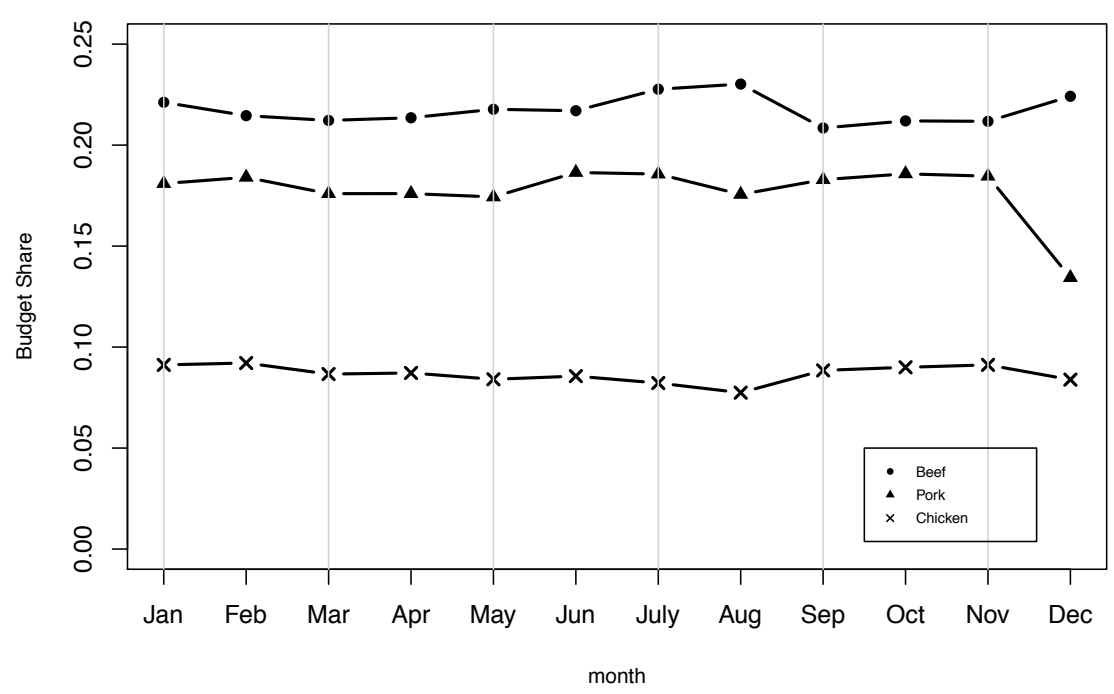

Figure 2: Average monthly budget shares of meat products in January 1998

- December 2000

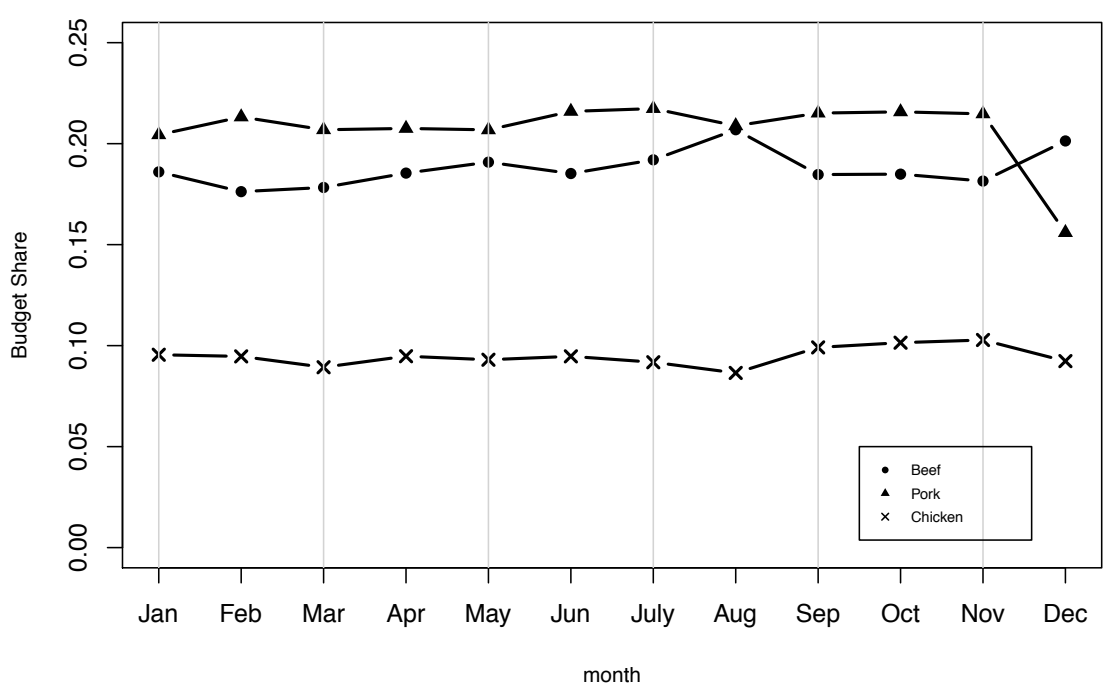

Figure 3: Average monthly budget shares of meat products in January 2003

- December 2006 
Table 1: Model selection

\begin{tabular}{c|cccrrr}
\hline \hline Model & Log-likelihood & \# of parameters & AIC & LR statics & d.f. & $\chi_{0.05}^{2}($ d.f. $)$ \\
\hline 1 & 1160.12 & 38 & -2244.24 & 211.95 & 21 & 32.67 \\
2 & 1188.27 & 44 & -2288.55 & 155.64 & 15 & 25.00 \\
3 & 1243.39 & 53 & -2380.77 & 45.41 & 13 & 12.59 \\
4 & 1266.09 & 59 & -2414.19 & - & 6 & - \\
\hline
\end{tabular}

1) AIC : -2 log-likelihood +2 ( \# of parameters )

2) LR statics : 2 ( log-likelihood(null model) - log-likelihood(alternative model) )

3) d.f. : degree of freedom

Next, we examine the structural change points in the Japanese meat market. Using the Hamilton filter, we calculate the conditional probability of each time point being at regime $s_{t}, \operatorname{Pr}\left(s_{t}=j \mid \boldsymbol{\Omega}_{t} ; \widehat{\boldsymbol{\Theta}}\right)(j=1,2)$ based on the data obtained through time $t, \Omega_{t}$ and set of estimated parameters $\widehat{\Theta}$ in MS-AIDS model. In Figure 4, we find that structure of budget shares is estimated to change almost instantaneously from regime 1 to regime 2 at the timing of first reported case of BSE on September 2001. However, we did not find any structural change points at the timing of bird flu, even when the number of regimes is increased from two to three. This result implies that there is no apparent effect on the consumers' behavior due to the bird flu in the Japanese meat market.

Using the conditional probability $\operatorname{Pr}\left(s_{t}=j \mid \boldsymbol{\Omega}_{t} ; \widehat{\boldsymbol{\Theta}}\right)$, we calculate the average budget share of $i_{t h}$ product at regime $s_{t}=j$ as

$$
\bar{w}_{i, s_{t}=j}=\frac{\sum_{t=1}^{T} \operatorname{Pr}\left(s_{t}=j \mid \boldsymbol{\Omega}_{t} ; \widehat{\boldsymbol{\Theta}}\right) \bar{w}_{i t}}{\sum_{t=1}^{T} \operatorname{Pr}\left(s_{t}=j \mid \boldsymbol{\Omega}_{t} ; \widehat{\boldsymbol{\Theta}}\right)} .
$$

Table 2 shows that beef and pork have significant changes between regime 1 and 2. Regime shift between regime 1 and regime 2 is considered due to the consumers' preference shift from beef to pork after the first BSE case. 


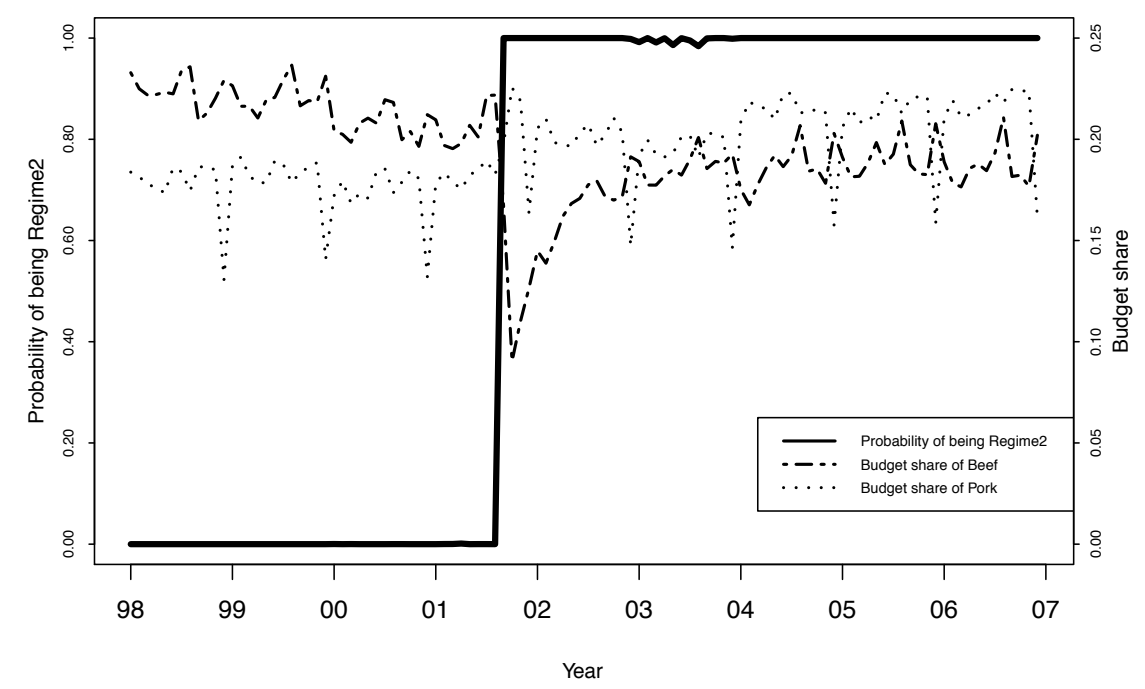

Figure 4: Probability of being Regime 2 and Budget share data of Beef and Pork

Table 2: Estimated average budget share

\begin{tabular}{c|cc}
\hline \hline & Regime1 & Regime2 \\
\hline Beef & 0.2155 & 0.1797 \\
Pork & 0.1781 & 0.2049 \\
Chicken & 0.0862 & 0.0955 \\
Fish & 0.5202 & 0.5199 \\
\hline
\end{tabular}


We show the estimates of parameters in Tables 3 and 4 . Table 3 shows that all parameters for prices $\left(\gamma_{i j}\right)$ except for the budget share of chicken relative to its own price are not significant. On the other hand, budget share of beef has a significant parameter for expenditure in regime 1 and the parameter for pork is significant in both regimes. These figures are used to calculate price and expenditure elasticities in Table 5.

Table 3: Estimated Parameters of MS-AIDS Model

\begin{tabular}{|c|c|c|c|c|c|c|c|c|c|}
\hline \multicolumn{5}{|c|}{ Regime1 } & \multicolumn{5}{|c|}{ Regime2 } \\
\hline & Estimate & Sd. Error & t-value & & & Estimate & Sd. Error & t-value & \\
\hline $\bar{\alpha}_{1}$ & 0.4359 & 0.1507 & 2.8920 & $* * *$ & $\bar{\alpha}_{1}$ & 0.2443 & 0.3114 & 0.7845 & \\
\hline $\bar{\alpha}_{2}$ & 0.4840 & 0.0754 & 6.4184 & $* * *$ & $\bar{\alpha}_{2}$ & 0.5429 & 0.1421 & 3.8199 & $* * *$ \\
\hline $\bar{\alpha}_{3}$ & 0.2873 & 0.0944 & 3.0430 & $* * *$ & $\bar{\alpha}_{3}$ & 0.2299 & 0.1074 & 2.1408 & $* *$ \\
\hline$\gamma_{11}$ & 0.0068 & 0.0657 & 0.1035 & & $\gamma_{11}$ & 0.0552 & 0.0802 & 0.6879 & \\
\hline$\gamma_{12}$ & -0.0174 & 0.0485 & -0.3596 & & $\gamma_{12}$ & -0.0197 & 0.0347 & -0.5677 & \\
\hline$\gamma_{13}$ & -0.0446 & 0.0384 & -1.1592 & & $\gamma_{13}$ & -0.0372 & 0.0277 & -1.3400 & \\
\hline$\gamma_{22}$ & 0.0523 & 0.0364 & 1.4362 & & $\gamma_{22}$ & 0.0378 & 0.0301 & 1.2575 & \\
\hline$\gamma_{23}$ & -0.0373 & 0.0348 & -1.0715 & & $\gamma_{23}$ & 0.0065 & 0.0264 & 0.2467 & \\
\hline$\gamma_{33}$ & 0.1042 & 0.0404 & 2.5807 & $* *$ & $\gamma_{33}$ & 0.0639 & 0.0297 & 2.1539 & $* *$ \\
\hline$\beta_{1}$ & -0.0672 & 0.0325 & -2.0659 & $* *$ & $\beta_{1}$ & -0.0404 & 0.0675 & -0.5980 & \\
\hline$\beta_{2}$ & -0.0687 & 0.0167 & -4.1228 & $* * *$ & $\beta_{2}$ & -0.0761 & 0.0303 & -2.5097 & $* *$ \\
\hline$\beta_{3}$ & -0.0126 & 0.0180 & -0.6970 & & $\beta_{3}$ & -0.0043 & 0.0231 & -0.1875 & \\
\hline
\end{tabular}

1) Significant level : $* * 5 \%, * * * 1 \%$

In Table 4, significantly negative trend effect in the budget share of beef is observed in regime 1 . We find that each of meat products has a different seasonal variation for August and December. The seasonal effects for August dummy variables are estimated to be significant for beef, while those for December dummy variables are not significant except pork. Furthermore, habit effects in the budget shares are estimated to be significant for beef and 
Table 4: Estimated Parameters in Intercept term

\begin{tabular}{|c|c|c|c|c|}
\hline & Estimate & Sd. Error & t-value & \\
\hline$\nu_{1}$ & -0.00039 & 0.00016 & -2.4463 & ** \\
\hline$\nu_{2}$ & -0.00001 & 0.00009 & -0.1515 & \\
\hline$\nu_{3}$ & -0.00006 & 0.00006 & -1.1065 & \\
\hline \multicolumn{5}{|c|}{ Seasonal (Aug) } \\
\hline & Estimate & Sd. Error & t-value & \\
\hline$\delta_{11}$ & 0.0115 & 0.0055 & 2.0894 & ** \\
\hline$\delta_{12}$ & -0.0048 & 0.0028 & -1.6942 & \\
\hline$\delta_{13}$ & -0.0049 & 0.0029 & -1.6673 & \\
\hline
\end{tabular}

\begin{tabular}{c|ccc} 
Trend (Regime 2) & \multicolumn{3}{l}{} \\
\hline & Estimate & Sd. Error & t-value \\
\hline$\nu_{1}$ & 0.00022 & 0.00032 & 0.6876 \\
$\nu_{2}$ & 0.00017 & 0.00016 & 1.0501 \\
$\nu_{3}$ & 0.00015 & 0.00011 & 1.4211 \\
\hline
\end{tabular}

\begin{tabular}{|c|c|c|c|c|}
\hline \multicolumn{5}{|c|}{ Habit effect } \\
\hline & Estimate & Sd. Error & t-value & \\
\hline$\phi_{11}$ & 0.1887 & 0.0709 & 2.6619 & $* *$ \\
\hline$\phi_{22}$ & 0.0440 & 0.0831 & 0.5294 & \\
\hline$\phi_{33}$ & 0.3939 & 0.0747 & 5.2704 & $* * *$ \\
\hline
\end{tabular}

\begin{tabular}{|c|c|c|c|c|}
\hline \multicolumn{5}{|c|}{ Seasonal (Dec) } \\
\hline & Estimate & Sd. Error & t-value & \\
\hline$\delta_{21}$ & 0.0186 & 0.0196 & 0.9480 & \\
\hline$\delta_{22}$ & -0.0254 & 0.0120 & -2.1287 & $* *$ \\
\hline$\delta_{23}$ & 0.0079 & 0.0124 & 0.6329 & \\
\hline \multicolumn{5}{|l|}{$\begin{array}{l}\text { Transition } \\
\text { probability }\end{array}$} \\
\hline & Estimate & Sd. Error & t-value & \\
\hline$\lambda_{11}$ & 4.3297 & 0.1427 & 30.3483 & $* * *$ \\
\hline$\lambda_{21}$ & -5.1701 & 1.1361 & -4.5509 & $* * *$ \\
\hline
\end{tabular}

1) Significant level : $* * 5 \%, * * * 1 \%$

chicken. This result means that the budget shares of beef and chicken for previous period have positive impacts on the current budget shares. Finally, Table 4 shows that estimated reparameterized transition probabilities $\lambda_{11}$ and $\lambda_{21}$ are significant, and transition probabilities $\pi_{11}$ and $\pi_{22}\left(=1-\pi_{21}\right)$ are estimated to be 0.987 and 0.994 . Therefore, structure of demand in the Japanese meat market tends to stay for a long time within the same regime.

Next, we consider the change of elasticities for beef and pork after the first BSE case. We do not compute elasticities for chicken primary because the price of chicken has changed very little over the study period, but also because we already found that substitution occurs mostly between beef and pork. Using the estimated parameters in Tables 3 and 4, we can calculate the Marshallian price elasticity $\eta_{i j, s_{t}}^{P}$ and expenditure elasticity $\eta_{i, s_{t}}^{E}$ at regime 
$s_{t}$ as

$$
\begin{aligned}
\eta_{i j, s_{t}}^{P} & =-\kappa_{i j}+\frac{\gamma_{i j, s_{t}}}{\bar{w}_{i, s_{t}}}-\frac{\beta_{i, s_{t}}}{\bar{w}_{i, s_{t}}}\left[\alpha_{j, s_{t}}+\sum_{k=1}^{N} \gamma_{k j, s_{t}} \log \bar{p}_{k, s_{t}}\right], \\
\eta_{i, s_{t}}^{E} & =\frac{\beta_{i, s_{t}}}{\bar{w}_{i, s_{t}}}+1
\end{aligned}
$$

where $\kappa_{i j}=1$ for $i=j$ and $\kappa_{i j}=0$ for $i \neq j$, and $\bar{p}_{k, s_{t}}$ is an average price at regime $s_{t}$. We also compute their associated standard errors via deltamethod. Table 5 shows the results of price and expenditure elasticities in regimes 1 and 2, respectively. The own-price elasticities of beef and pork

Table 5: Price elasticities and Expenditure elasticities

\begin{tabular}{c|cccccc} 
Regime 1 & \multicolumn{3}{c}{ Price $\left(\eta_{i j}^{P}\right)$} & \multicolumn{3}{c}{ Expenditure $\left(\eta_{i}^{E}\right)$} \\
\hline \hline \multirow{3}{*}{ Beef } & Beef & \multicolumn{3}{c}{ Pork } & & \\
& -0.815 & $* *$ & 0.062 & 0.688 & $* * *$ \\
& $(-2.311)$ & & $(0.227)$ & $(3.801)$ & \\
Pork & 0.091 & & -0.529 & $* * *$ & 0.615 & $* * *$ \\
& $(0.384)$ & & $(-3.143)$ & & $(7.563)$ & \\
\hline
\end{tabular}

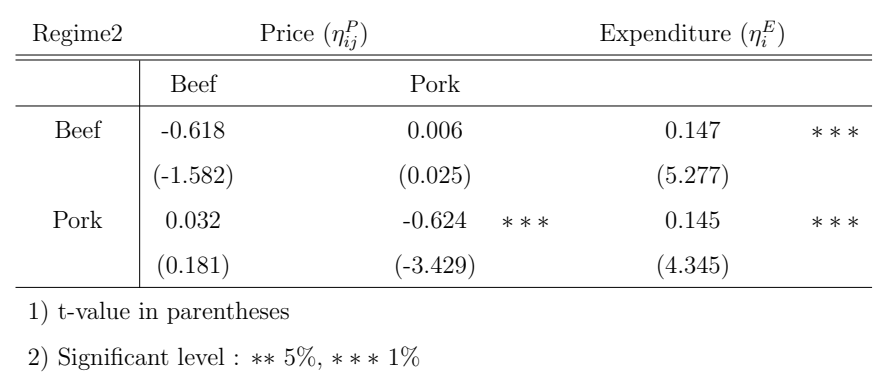

in regime 1 are significantly negative, but beef price elasticity in regime 2 is not significant. From the estimated cross-price elasticities, no statistically significant price-driven substitution occurred between beef and pork in both regimes. Based on the estimated expenditure elasticities, we find that consumptions of beef and pork are responsive to the change in total expenditure on meat products in both regimes. 


\section{Conclusion and Discussion}

Previous studies such as Peterson and Chen (2005) and Ishida et al. (2006, 2010) assumed that starting-points of structural shift in demands are known. For example, Ishida et al. $(2006,2010)$ assumed that there are two structural shifts, the first at the timing of the first reported cases of BSE in September 2001 and the second at the first reported case of bird flu in January 2004, both in Japan. However such structural shifts, if any, ought to be reflected in the demand data itself. Thus, in this study, we applied the Markovswitching almost ideal demand system (MS-AIDS) model proposed by Allais and Nichèle (2007) to uncover the structural change points in the Japanese meat market.

First, we illustrate that MS-AIDS model is able to identify the timing of the first reported case of BSE in September 2001 as the structural change point, but the same model does not recognize the timing of the first bird flu. This result shows that there is no apparent impact of bird flu on the Japanese consumers' meat consumption. Regime 1 before the first BSE is characterized by higher beef budget share relative to the pork budget share, while regime 2 is characterized by the reversal of budget shares between beef and pork (see Table 2). The first BSE changed consumers' preference away from beef to pork.

Next, we find that own-price elasticity of beef in regime 1 is significantly negative, but that in regime 2 is no longer significant. This shift in regime 2 is the reflection of the fact that food safety on beef becomes more important than its price to Japanese consumers. There were two streams of events that were likely to have contributed to this heightened awareness of safety of beef products, one domestic, and the other international.

Domestically, in October 18, 2001, a month after the first reported BSE 
case in Japan, the Japanese government started complete survey of domestic cattle. At the same time the government instituted buyback program of domestic beef processed before that date. A well known food importer/producer Snow Brand Food Company disguised imported beef as domestic beef to illegally take advantage of the government buyback program. It was since revealed that many other major meat companies includes Nippon Ham committed the same subsidy fraud. As a result consumers have become weary of beef in general.

Internationally, the first BSE case in the U.S., the largest or second largest beef exporter to Japan during 2001-2003, was confirmed on December 2003. The Japanese government immediately announced a ban on import of American beef. On December 2005 the Japanese government resumed importing American beef under stricter conditions. Nevertheless banned specified-risk materials of beef products were found from the imported beef from the U.S. since January 2006.

Finally, we note some issues which are left for future work. This study does not establish direct relationship between the regime shift of meat demands and the consumers' awareness of risk of vCJD and bird flu, though the regime shift was found to coincide with the first BSE case timewise. This awareness itself could be affected by consumers demographics such as age, composition of the family, household income (Adda, 2007). In addition, consumers' choice of meat products could be influenced by the country-oforigin information (Peterson and Chen, 2005) and by the mass media such as news papers and TV (Verbeke and Ward, 2001). Therefore, future research is needed for integration of theses effects into the Markov-switching almost ideal demand system model. 


\section{References}

Adda, J. (2007) 'Behavior towards health risks: An empirical study using the "mad cow" crisis as an experiment.' Journal of Risk and Uncertainty $35(3), 285-305$

Allais, O., and V. Nichèle (2007) 'Capturing structural changes in french meat and fish demand over the period 1991 2002.' European review of agricultural economics

Banks, J., R. Blundell, and A. Lewbel (1997) 'Quadratic engel curves and consumer demand.' Review of Economics and Statistics 79(4), 527-539

Berndt, E. K., B. H. Hall, R. E. Hall, and J. A. Hausman (1974) 'Estimation and inference in nonlinear structural models.' Annals of Economic and Social Measurement 3, 653-665

Bickel, P.J., Y. Ritovand, and T. Ryden (1998) 'Asymptotic normality of the maximum-likelihood estimator for general hidden markov models.' Annals of Statistics 26(4), 1614-1635

Christensen, L. R., D. W. Jorgenson, and L. J. Lau (1975) 'Transcendental logarithmic utility functions.' American Economic Review 65(3), 367-383

Cooper, R. J., and K. R. McLaren (1992) 'An empirically oriented demand system with improved regularity properties.' Canadian Journal of Economics 25(3), 652-668

Deaton, A., and J. Muellbauer (1980) 'An almost ideal demand system.' The American Economic Review 70(3), 312-326

Garcia, R. (1998) 'Asymptotic null distribution of the likelihood ratio test in markov switching models.' International Economic Review pp. 763-788 
Hamilton, J. D. (1989) 'A new approach to the economic analysis of nonstationary time series and the business cycle.' Econometrica: Journal of the Econometric Society 57(2), 357-384

Ishida, T., N. Ishikawa, and M. Fukushige (2006) 'Impact of bse and bird flu on consumers' meat demand in japan.' DISCUSSION PAPER SERIES in Osaka university

Ishida, T., N. Ishikawa, and M. Fukushige (2010) 'Impact of bse and bird flu on consumers' meat demand in japan.' Applied Economics 42(1), 49-56

Jin, H. J., and W. W. Koo (2003) 'The effect of the bse outbreak in japan on consumers' preferences.' European Review of Agricultural Economics 30(2), 173-192

Moosa, I. A., and J. L. Baxter (2002) 'Modeling the trend and seasonal within an aids model of demand for alcoholic beverage in the united kingdom.' Journal of Applied Econometrics 17(2), 95-106

Ohtani, K., and S. Katayama (1986) 'A gradual switching regression model with autocorrelated errors.' Economics Letters 21(2), 169-172

Peterson, H. H., and Y. J. K. Chen (2005) 'The impact of bse on japanese retail meat demand.' Agribusiness 21(3), 313-327

Rickertsen, K. (1996) 'Structural change and the demand for meat and fish in norway.' European Review of Agricultural Economics 23(3), 316

Theil, H. (1965) 'The information approach to demand analysis.' Econometrica $33(1), 67-87$ 
Verbeke, W., and R. W. Ward (2001) 'A fresh meat almost ideal demand system incorporating negative tv press and advertising impact.' Agricultural Economics 25(2-3), 359-374

\section{A Log-likelihood function of MS-AIDS model}

Let $\boldsymbol{w}_{t}$ be a $(N-1) \times 1$ vector of budget shares at time $t, \bar{w}_{i t}(i=1,2, \ldots, N-$ 1) and $\boldsymbol{x}_{t}$ be a vector of explanatory variables. We define a parameter vector in regime $s_{t}$ as $\boldsymbol{\theta}_{s_{t}}$ and error term of MS-AIDS model as $\boldsymbol{\varepsilon}_{t}=\left[\varepsilon_{1 t}, \varepsilon_{2 t}, \ldots, \varepsilon_{N-1, t}\right]^{\prime} \sim$ $\mathcal{N}\left(\mathbf{0}, \boldsymbol{\Sigma}_{s_{t}}\right)$ with a $(N-1) \times(N-1)$ variance-covariance matrix $\boldsymbol{\Sigma}_{s_{t}}$.

Suppose that distribution of $\boldsymbol{w}_{t}$ conditional on $\boldsymbol{x}_{t}, s_{t}$ and $\boldsymbol{\theta}=\left[\boldsymbol{\theta}_{1}, \ldots, \boldsymbol{\theta}_{K}\right.$, $\left.\boldsymbol{\Sigma}_{1}, \ldots, \boldsymbol{\Sigma}_{K}\right]^{\prime}$ is defined as $p\left(\boldsymbol{w}_{t} \mid \boldsymbol{x}_{t}, s_{t} ; \boldsymbol{\theta}\right)$ and let $\boldsymbol{\pi}$ be a vector of transition probabilities $\pi_{i j}, i=1,2, \ldots, K, j=1,2, \ldots, K-1$. Then the conditional $\log$-likelihood function with respect to parameter set $\boldsymbol{\Theta} \equiv\{\boldsymbol{\theta}, \boldsymbol{\pi}\}$ under all the observations $\left(\boldsymbol{w}_{t}, \boldsymbol{x}_{t}\right), t=1,2, \ldots, T$ is

$$
\begin{aligned}
\ell(\boldsymbol{\Theta}) & =\sum_{t=1}^{T} \log \left[\sum_{s_{t}=1}^{K} p\left(\boldsymbol{w}_{t} \mid \boldsymbol{x}_{t}, s_{t} ; \boldsymbol{\theta}\right) \operatorname{Pr}\left(s_{t} \mid \boldsymbol{\Omega}_{t-1} ; \boldsymbol{\Theta}\right)\right] \\
& =\sum_{t=1}^{T} \log p\left(\boldsymbol{w}_{t} \mid \mathcal{Z}_{t} ; \boldsymbol{\Theta}\right)
\end{aligned}
$$

where $\boldsymbol{\Omega}_{t}$ is an information set containing all observations obtained through time $t: \boldsymbol{\Omega}_{t} \equiv\left\{\boldsymbol{w}_{t}, \boldsymbol{w}_{t-1}, \ldots, \boldsymbol{w}_{1}, \boldsymbol{x}_{t}, \boldsymbol{x}_{t-1}, \ldots, \boldsymbol{x}_{1}\right\}$ and $\mathcal{Z}_{t}$ is an information set of lags of $\boldsymbol{w}_{t}$ and observable explanatory variables obtained through time $t: \mathcal{Z}_{t} \equiv\left\{\boldsymbol{w}_{t-1}, \boldsymbol{w}_{t-2}, \ldots, \boldsymbol{w}_{1}, \boldsymbol{x}_{t}, \boldsymbol{x}_{t-1}, \ldots, \boldsymbol{x}_{1}\right\}$. The conditional probability $\operatorname{Pr}\left(s_{t} \mid \boldsymbol{\Omega}_{t} ; \boldsymbol{\Theta}\right)$ represents the probability about the value of $s_{t}$ based on data obtained through time $t$ and based on parameter set $\Theta$.

The maximum likelihood estimator of variance-covariance matrix $\boldsymbol{\Sigma}_{s_{t}}$ is derived as follows: From (A.1) and (A.2), a first derivative of log-likelihood 
function with respect to inverse variance-covariance matrix $\Sigma_{s_{t}}^{-1}$ of regime $s_{t}=j$ is

$$
\begin{aligned}
& \frac{\partial \ell(\boldsymbol{\Theta})}{\partial \boldsymbol{\Sigma}_{s_{t}=j}^{-1}} \\
& =\sum_{t} \frac{1}{\sum_{s_{t}} p\left(\boldsymbol{w}_{t} \mid \boldsymbol{x}_{t}, s_{t} ; \boldsymbol{\theta}\right) \operatorname{Pr}\left(s_{t} \mid \boldsymbol{\Omega}_{t-1} ; \boldsymbol{\theta}, \boldsymbol{\pi}\right)} \frac{\partial \sum_{s_{t}} p\left(\boldsymbol{w}_{t} \mid \boldsymbol{x}_{t}, s_{t} ; \boldsymbol{\theta}\right) \operatorname{Pr}\left(s_{t} \mid \boldsymbol{\Omega}_{t-1} ; \boldsymbol{\theta}, \boldsymbol{\pi}\right)}{\partial \boldsymbol{\Sigma}_{s_{t}=j}^{-1}} \\
& =\sum_{t} \frac{1}{\sum_{s_{t}} p\left(\boldsymbol{w}_{t} \mid \boldsymbol{x}_{t}, s_{t} ; \boldsymbol{\theta}\right) \operatorname{Pr}\left(s_{t} \mid \boldsymbol{\Omega}_{t-1} ; \boldsymbol{\theta}, \boldsymbol{\pi}\right)} \frac{\partial p\left(\boldsymbol{w}_{t} \mid \boldsymbol{x}_{t}, s_{t}=j ; \boldsymbol{\theta}\right) \operatorname{Pr}\left(s_{t}=j \mid \boldsymbol{\Omega}_{t-1} ; \boldsymbol{\theta}, \boldsymbol{\pi}\right)}{\partial \boldsymbol{\Sigma}_{s_{t}=j}^{-1}} \\
& =\sum_{t} \frac{p\left(\boldsymbol{w}_{t} \mid \boldsymbol{x}_{t}, s_{t}=j ; \boldsymbol{\theta}\right) \operatorname{Pr}\left(s_{t}=j \mid \boldsymbol{\Omega}_{t-1} ; \boldsymbol{\theta}, \boldsymbol{\pi}\right)}{\sum_{s_{t}} p\left(\boldsymbol{w}_{t} \mid \boldsymbol{x}_{t}, s_{t} ; \boldsymbol{\theta}\right) \operatorname{Pr}\left(s_{t} \mid \boldsymbol{\Omega}_{t-1} ; \boldsymbol{\theta}, \boldsymbol{\pi}\right)} \frac{\partial \log \left[p\left(\boldsymbol{w}_{t} \mid \boldsymbol{x}_{t}, s_{t}=j ; \boldsymbol{\theta}\right) \operatorname{Pr}\left(s_{t}=j \mid \boldsymbol{\Omega}_{t-1} ; \boldsymbol{\theta}, \boldsymbol{\pi}\right)\right]}{\partial \boldsymbol{\Sigma}_{s_{t}=j}^{-1}} \\
& =\sum_{t} \operatorname{Pr}\left(s_{t}=j \mid \boldsymbol{\Omega}_{t} ; \boldsymbol{\Theta}\right) \frac{\partial \log p\left(\boldsymbol{w}_{t} \mid \boldsymbol{x}_{t}, s_{t}=j ; \boldsymbol{\theta}\right)}{\partial \boldsymbol{\Sigma}_{s_{t}=j}^{-1}} .
\end{aligned}
$$

Assuming that

$$
p\left(\boldsymbol{w}_{t} \mid \boldsymbol{x}_{t}, s_{t} ; \boldsymbol{\theta}\right)=(2 \pi)^{-\frac{N-1}{2}}\left|\boldsymbol{\Sigma}_{s_{t}}\right|^{-\frac{1}{2}} \exp \left(-\frac{1}{2} \boldsymbol{\varepsilon}_{t}^{\prime} \boldsymbol{\Sigma}_{s_{t}}^{-1} \boldsymbol{\varepsilon}_{t}\right),
$$

we have

$$
\begin{aligned}
\frac{\partial \log p\left(\boldsymbol{w}_{t} \mid \boldsymbol{x}_{t}, s_{t}=j ; \boldsymbol{\theta}\right)}{\partial \boldsymbol{\Sigma}_{s_{t}=j}^{-1}} & =\frac{\partial}{\partial \boldsymbol{\Sigma}_{s_{t}=j}^{-1}}\left[-\frac{N-1}{2} \log (2 \pi)+\frac{1}{2} \log \left|\boldsymbol{\Sigma}_{s_{t}}\right|^{-1}-\frac{1}{2} \varepsilon_{t}^{\prime} \boldsymbol{\Sigma}_{s_{t}}^{-1} \boldsymbol{\varepsilon}_{t}\right] \\
& =\frac{1}{2} \frac{\partial \log \left|\boldsymbol{\Sigma}_{s_{t}=j}\right|^{-1}}{\partial \boldsymbol{\Sigma}_{s_{t}=j}^{-1}}-\frac{1}{2} \frac{\partial\left(\boldsymbol{\varepsilon}_{t}^{\prime} \boldsymbol{\Sigma}_{s_{t}=j}^{-1} \boldsymbol{\varepsilon}_{t}\right)}{\partial \boldsymbol{\Sigma}_{s_{t}=j}^{-1}} \\
& =\frac{1}{2} \frac{\partial \log \left|\boldsymbol{\Sigma}_{s_{t}=j}^{-1}\right|}{\partial \boldsymbol{\Sigma}_{s_{t}=j}^{-1}}-\frac{1}{2} \frac{\partial \operatorname{tr}\left\{\boldsymbol{\Sigma}_{s_{t}=j}^{-1} \boldsymbol{\varepsilon}_{t} \boldsymbol{\varepsilon}_{t}^{\prime}\right\}}{\partial \boldsymbol{\Sigma}_{s_{t}=j}^{-1}} \\
& =\frac{1}{2} \boldsymbol{\Sigma}_{s_{t}=j}-\frac{1}{2} \boldsymbol{\varepsilon}_{t} \boldsymbol{\varepsilon}_{t}^{\prime}
\end{aligned}
$$

and

$$
\frac{\partial \ell(\boldsymbol{\Theta})}{\partial \boldsymbol{\Sigma}_{s_{t}=j}^{-1}}=\sum_{t=1}^{T} \operatorname{Pr}\left(s_{t}=j \mid \boldsymbol{\Omega}_{t} ; \boldsymbol{\Theta}\right)\left[\frac{1}{2} \boldsymbol{\Sigma}_{s_{t}=j}-\frac{1}{2} \boldsymbol{\varepsilon}_{t} \boldsymbol{\varepsilon}_{t}^{\prime}\right]=\mathbf{0} .
$$

Therefore we have

$$
\widehat{\boldsymbol{\Sigma}}_{s_{t}=j}=\frac{\sum_{t=1}^{T} \operatorname{Pr}\left(s_{t}=j \mid \boldsymbol{\Omega}_{t} ; \boldsymbol{\Theta}\right) \hat{\boldsymbol{\varepsilon}}_{t} \hat{\varepsilon}_{t}^{\prime}}{\sum_{t=1}^{T} \operatorname{Pr}\left(s_{t}=j \mid \boldsymbol{\Omega}_{t} ; \boldsymbol{\Theta}\right)} .
$$




\section{B Calculation of score function}

\section{Score function with respect to $\theta$}

The first derivative of $\log$-likelihood with respect to $\boldsymbol{\theta}$ is

$$
\begin{aligned}
& \frac{\partial \log p\left(\boldsymbol{w}_{t} \mid \mathcal{Z}_{t} ; \boldsymbol{\Theta}\right)}{\partial \boldsymbol{\theta}} \\
& =\sum_{s_{t}=1}^{K} \frac{\partial \log p\left(\boldsymbol{w}_{t} \mid \boldsymbol{x}_{t}, s_{t} ; \boldsymbol{\theta}\right)}{\partial \boldsymbol{\theta}} \operatorname{Pr}\left(s_{t} \mid \boldsymbol{\Omega}_{t} ; \boldsymbol{\Theta}\right) \\
& +\sum_{\tau=1}^{t-1} \sum_{s_{\tau}=1}^{K} \frac{\partial \log p\left(\boldsymbol{w}_{\tau} \mid \boldsymbol{x}_{\tau}, s_{\tau} ; \boldsymbol{\theta}\right)}{\partial \boldsymbol{\theta}}\left\{\operatorname{Pr}\left(s_{\tau} \mid \boldsymbol{\Omega}_{t} ; \boldsymbol{\Theta}\right)-\operatorname{Pr}\left(s_{\tau} \mid \boldsymbol{\Omega}_{t-1} ; \boldsymbol{\Theta}\right)\right\}
\end{aligned}
$$

for $t=2,3, \ldots, T$ and

$$
\frac{\partial \log p\left(\boldsymbol{w}_{1} \mid \mathcal{Z}_{1} ; \boldsymbol{\Theta}\right)}{\partial \boldsymbol{\theta}}=\sum_{s_{1}=1}^{K} \frac{\partial \log p\left(\boldsymbol{w}_{1} \mid \boldsymbol{x}_{1}, s_{1} ; \boldsymbol{\theta}\right)}{\partial \boldsymbol{\theta}} \operatorname{Pr}\left(s_{1} \mid \boldsymbol{\Omega}_{1} ; \boldsymbol{\Theta}\right) .
$$

\section{Score function with respect to $\pi_{i j}$}

The first derivative of log-likelihood with respect to the transition probabilities $\pi_{i j}, i=1,2, \ldots, K, j=1,2, \ldots, K-1$ is

$$
\begin{aligned}
& \frac{\partial \log p\left(\boldsymbol{w}_{t} \mid \mathcal{Z}_{t} ; \boldsymbol{\Theta}\right)}{\partial \pi_{i j}} \\
& =\pi_{i j}^{-1} \operatorname{Pr}\left(s_{t}=j, s_{t-1}=i \mid \boldsymbol{\Omega}_{t} ; \boldsymbol{\Theta}\right)-\pi_{i K}^{-1} \operatorname{Pr}\left(s_{t}=K, s_{t-1}=i \mid \boldsymbol{\Omega}_{t} ; \boldsymbol{\Theta}\right) \\
& +\pi_{i j}^{-1} \sum_{\tau=2}^{t-1}\left[\operatorname{Pr}\left(s_{\tau}=j, s_{\tau-1}=i \mid \boldsymbol{\Omega}_{t} ; \boldsymbol{\Theta}\right)-\operatorname{Pr}\left(s_{\tau}=j, s_{\tau-1}=i \mid \boldsymbol{\Omega}_{t-1} ; \boldsymbol{\Theta}\right)\right] \\
& -\pi_{i K}^{-1} \sum_{\tau=2}^{t-1}\left[\operatorname{Pr}\left(s_{\tau}=K, s_{\tau-1}=i \mid \boldsymbol{\Omega}_{t} ; \boldsymbol{\Theta}\right)-\operatorname{Pr}\left(s_{\tau}=K, s_{\tau-1}=i \mid \boldsymbol{\Omega}_{t-1} ; \boldsymbol{\Theta}\right)\right] \\
& +\sum_{s_{1}=1}^{K} \frac{\partial \log \operatorname{Pr}\left(s_{1} ; \boldsymbol{\pi}\right)}{\partial \pi_{i j}}\left[\operatorname{Pr}\left(s_{1} \mid \boldsymbol{\Omega}_{t} ; \boldsymbol{\Theta}\right)-\operatorname{Pr}\left(s_{1} \mid \boldsymbol{\Omega}_{t-1} ; \boldsymbol{\Theta}\right)\right]
\end{aligned}
$$

for $i=1,2, \ldots, K, j=1,2, \ldots, K-1$ and $t=2,3, \ldots, T$, and when $t=1$,

$$
\frac{\partial \log p\left(\boldsymbol{w}_{1} \mid \mathcal{Z}_{1} ; \boldsymbol{\Theta}\right)}{\partial \pi_{i j}}=\sum_{s_{1}=1}^{K} \frac{\partial \log \operatorname{Pr}\left(s_{1} ; \boldsymbol{\pi}\right)}{\partial \pi_{i j}} \operatorname{Pr}\left(s_{1} \mid \boldsymbol{\Omega}_{1} ; \boldsymbol{\Theta}\right) .
$$


To satisfy $0 \leq \pi_{i j} \leq 1$ and $\sum_{j=1}^{K} \pi_{i j}=1$, we reparameterize the transition probability $\pi_{i j}$ as follows

$$
\lambda_{i j}=\log \left(\pi_{i j} / \pi_{i K}\right), \quad i=1,2, \ldots, K, \quad j=1,2, \ldots, K-1
$$

and estimate the parameter $\lambda_{i j}$ instead of the transition probability $\pi_{i j}$.

To calculate the score function with respect to the parameter $\lambda_{i j}$, we apply the chain rule as follows

$$
\frac{\partial \log p\left(\boldsymbol{w}_{t} \mid \mathcal{Z}_{t} ; \boldsymbol{\Theta}\right)}{\partial \lambda_{i j}}=\frac{\partial \log p\left(\boldsymbol{w}_{t} \mid \mathcal{Z}_{t} ; \boldsymbol{\Theta}\right)}{\partial \pi_{i j}} \times \frac{\partial \pi_{i j}}{\partial \lambda_{i j}}
$$

and the partial derivative of $\pi_{i j}$ with respect to $\lambda_{i j}$ is obtained from

$$
\begin{aligned}
\frac{\partial \lambda_{i j}}{\partial \pi_{i j}} & =\frac{\partial}{\partial \pi_{i j}} \log \left(\pi_{i j} / \pi_{i K}\right) \\
& =\frac{\partial \log \left(\pi_{i j}\right)}{\partial \pi_{i j}}-\frac{\partial \log \left(\pi_{i K}\right)}{\partial \pi_{i j}} \\
& =\frac{\partial \log \left(\pi_{i j}\right)}{\partial \pi_{i j}}-\frac{\partial \log \left(\pi_{i K}\right)}{\partial \pi_{i K}} \times \frac{\partial \pi_{i K}}{\partial \pi_{i j}} \\
& =\frac{1}{\pi_{i j}}+\frac{1}{\pi_{i K}},
\end{aligned}
$$

where $i=1,2, \ldots, K, j=1,2, \ldots, K-1$.

\section{Calculation of joint probabilities}

We need to assess the changes in the probabilities $\operatorname{Pr}\left(s_{\tau} \mid \boldsymbol{\Omega}_{t} ; \boldsymbol{\Theta}\right)-\operatorname{Pr}\left(s_{\tau} \mid \boldsymbol{\Omega}_{t-1} ; \boldsymbol{\Theta}\right)$ in (B.1) and $\operatorname{Pr}\left(s_{\tau}, s_{\tau-1} \mid \boldsymbol{\Omega}_{t} ; \boldsymbol{\Theta}\right)-\operatorname{Pr}\left(s_{\tau}, s_{\tau-1} \mid \boldsymbol{\Omega}_{t-1} ; \boldsymbol{\Theta}\right)$ in (B.3). With joint probabilities $\operatorname{Pr}\left(s_{t-1}, s_{\tau}, s_{\tau-1} \mid \boldsymbol{\Omega}_{t-1} ; \boldsymbol{\Theta}\right)$ and $\operatorname{Pr}\left(s_{t}, s_{\tau}, s_{\tau-1} \mid \boldsymbol{\Omega}_{t} ; \boldsymbol{\Theta}\right)$, the changes in the probabilities $\operatorname{Pr}\left(s_{\tau} \mid \boldsymbol{\Omega}_{t} ; \boldsymbol{\Theta}\right)-\operatorname{Pr}\left(s_{\tau} \mid \boldsymbol{\Omega}_{t-1} ; \boldsymbol{\Theta}\right)$ and $\operatorname{Pr}\left(s_{\tau}, s_{\tau-1} \mid \boldsymbol{\Omega}_{t} ; \boldsymbol{\Theta}\right)-$ $\operatorname{Pr}\left(s_{\tau}, s_{\tau-1} \mid \boldsymbol{\Omega}_{t-1} ; \boldsymbol{\Theta}\right)$ are calculated as

$$
\operatorname{Pr}\left(s_{\tau}, s_{\tau-1} \mid \boldsymbol{\Omega}_{t} ; \boldsymbol{\Theta}\right)-\operatorname{Pr}\left(s_{\tau}, s_{\tau-1} \mid \boldsymbol{\Omega}_{t-1} ; \boldsymbol{\Theta}\right)
$$




$$
=\sum_{s_{t}} \operatorname{Pr}\left(s_{t}, s_{\tau}, s_{\tau-1} \mid \boldsymbol{\Omega}_{t} ; \boldsymbol{\Theta}\right)-\sum_{s_{t-1}} \operatorname{Pr}\left(s_{t-1}, s_{\tau}, s_{\tau-1} \mid \boldsymbol{\Omega}_{t-1} ; \boldsymbol{\Theta}\right)
$$

and

$$
\begin{aligned}
& \operatorname{Pr}\left(s_{\tau} \mid \boldsymbol{\Omega}_{t} ; \boldsymbol{\Theta}\right)-\operatorname{Pr}\left(s_{\tau} \mid \boldsymbol{\Omega}_{t-1} ; \boldsymbol{\Theta}\right) \\
& =\sum_{s_{\tau-1}} \operatorname{Pr}\left(s_{\tau}, s_{\tau-1} \mid \boldsymbol{\Omega}_{t} ; \boldsymbol{\Theta}\right)-\sum_{s_{\tau-1}} \operatorname{Pr}\left(s_{\tau}, s_{\tau-1} \mid \boldsymbol{\Omega}_{t-1} ; \boldsymbol{\Theta}\right) .
\end{aligned}
$$

The derivation of joint probabilities $\operatorname{Pr}\left(s_{t-1}, s_{\tau}, s_{\tau-1} \mid \boldsymbol{\Omega}_{t-1} ; \boldsymbol{\Theta}\right)$ and $\operatorname{Pr}\left(s_{t}, s_{\tau}, s_{\tau-1} \mid \boldsymbol{\Omega}_{t} ; \boldsymbol{\Theta}\right)$ is as shown below.

Suppose that we have a joint probability for regimes $s_{t-1}, s_{\tau}$ and $s_{\tau-1}$ :

$$
\operatorname{Pr}\left(s_{t-1}, s_{\tau}, s_{\tau-1} \mid \boldsymbol{\Omega}_{t-1} ; \boldsymbol{\Theta}\right)
$$

for $\tau<t$, a joint probability $\operatorname{Pr}\left(s_{t}, s_{\tau}, s_{\tau-1} \mid \boldsymbol{\Omega}_{t} ; \boldsymbol{\Theta}\right)$ is calculated as follows

$$
\begin{aligned}
& \operatorname{Pr}\left(s_{t}, s_{\tau}, s_{\tau-1} \mid \boldsymbol{\Omega}_{t} ; \boldsymbol{\Theta}\right)=\operatorname{Pr}\left(s_{t}, s_{\tau}, s_{\tau-1} \mid \boldsymbol{w}_{t}, \boldsymbol{x}_{t}, \boldsymbol{\Omega}_{t-1} ; \boldsymbol{\Theta}\right) \\
& =\frac{g\left(s_{t}, s_{\tau}, s_{\tau-1}, \boldsymbol{w}_{t} \mid \boldsymbol{x}_{t}, \boldsymbol{\Omega}_{t-1} ; \boldsymbol{\Theta}\right)}{p\left(\boldsymbol{w}_{t} \mid \boldsymbol{x}_{t}, \boldsymbol{\Omega}_{t-1} ; \boldsymbol{\Theta}\right)}=\frac{\sum_{s_{t-1}} g\left(s_{t-1}, s_{t}, s_{\tau}, s_{\tau-1}, \boldsymbol{w}_{t} \mid \boldsymbol{x}_{t}, \boldsymbol{\Omega}_{t-1} ; \boldsymbol{\Theta}\right)}{p\left(\boldsymbol{w}_{t} \mid \boldsymbol{x}_{t}, \boldsymbol{\Omega}_{t-1} ; \boldsymbol{\Theta}\right)} \\
& =\frac{\sum_{s_{t-1}} g\left(s_{t-1}, s_{t}, s_{\tau}, s_{\tau-1}, \boldsymbol{w}_{t} \mid \boldsymbol{x}_{t}, \boldsymbol{\Omega}_{t-1} ; \boldsymbol{\Theta}\right)}{\sum_{s_{t}} \sum_{s_{t-1}} \sum_{s_{\tau}} \sum_{s_{\tau-1}} g\left(s_{t-1}, s_{t}, s_{\tau}, s_{\tau-1}, \boldsymbol{w}_{t} \mid \boldsymbol{x}_{t}, \boldsymbol{\Omega}_{t-1} ; \boldsymbol{\Theta}\right)} \\
& =\frac{\sum_{s_{t-1}} \operatorname{Pr}\left(s_{t-1}, s_{t}, s_{\tau}, s_{\tau-1} \mid \boldsymbol{\Omega}_{t-1} ; \boldsymbol{\Theta}\right) p\left(\boldsymbol{w}_{t} \mid \boldsymbol{x}_{t}, s_{t-1}, s_{t}, s_{\tau}, s_{\tau-1} ; \boldsymbol{\Theta}\right)}{\sum_{s_{t}} \sum_{s_{t-1}} \sum_{s_{\tau}} \sum_{s_{\tau-1}} \operatorname{Pr}\left(s_{t-1}, s_{t}, s_{\tau}, s_{\tau-1} \mid \boldsymbol{\Omega}_{t-1} ; \boldsymbol{\Theta}\right) p\left(\boldsymbol{w}_{t} \mid \boldsymbol{x}_{t}, s_{t-1}, s_{t}, s_{\tau}, s_{\tau-1} ; \boldsymbol{\Theta}\right)}
\end{aligned}
$$

Since the transition is Markovian

$$
\begin{aligned}
\operatorname{Pr}\left(s_{t-1}, s_{t}, s_{\tau}, s_{\tau-1} \mid \boldsymbol{\Omega}_{t-1} ; \boldsymbol{\Theta}\right) & =\operatorname{Pr}\left(s_{t} \mid s_{t-1}, s_{\tau}, s_{\tau-1}\right) \operatorname{Pr}\left(s_{t-1}, s_{\tau}, s_{\tau-1} \mid \boldsymbol{\Omega}_{t-1} ; \boldsymbol{\Theta}\right) \\
& =\operatorname{Pr}\left(s_{t} \mid s_{t-1}\right) \operatorname{Pr}\left(s_{t-1}, s_{\tau}, s_{\tau-1} \mid \boldsymbol{\Omega}_{t-1} ; \boldsymbol{\Theta}\right)
\end{aligned}
$$

and we have

$$
p\left(\boldsymbol{w}_{t} \mid \boldsymbol{x}_{t}, s_{t-1}, s_{t}, s_{\tau}, s_{\tau-1} ; \boldsymbol{\Theta}\right)=p\left(\boldsymbol{w}_{t} \mid \boldsymbol{x}_{t}, s_{t} ; \boldsymbol{\Theta}\right),
$$


(C.4) can be rewritten as

$$
\begin{aligned}
& \operatorname{Pr}\left(s_{t}, s_{\tau}, s_{\tau-1} \mid \boldsymbol{\Omega}_{t} ; \boldsymbol{\Theta}\right) \\
& =\frac{\sum_{s_{t-1}} \operatorname{Pr}\left(s_{t} \mid s_{t-1}\right) \operatorname{Pr}\left(s_{t-1}, s_{\tau}, s_{\tau-1} \mid \boldsymbol{\Omega}_{t-1} ; \boldsymbol{\Theta}\right) p\left(\boldsymbol{w}_{t} \mid \boldsymbol{x}_{t}, s_{t} ; \boldsymbol{\Theta}\right)}{\sum_{s_{t}} \sum_{s_{t-1}} \sum_{s_{\tau}} \sum_{s_{\tau-1}} \operatorname{Pr}\left(s_{t} \mid s_{t-1}\right) \operatorname{Pr}\left(s_{t-1}, s_{\tau}, s_{\tau-1} \mid \boldsymbol{\Omega}_{t-1} ; \boldsymbol{\Theta}\right) p\left(\boldsymbol{w}_{t} \mid \boldsymbol{x}_{t}, s_{t} ; \boldsymbol{\Theta}\right)}
\end{aligned}
$$

where $\operatorname{Pr}\left(s_{t} \mid s_{t-1}\right)$ is the transition probability and $p\left(\boldsymbol{w}_{t} \mid \boldsymbol{x}_{t}, s_{t} ; \boldsymbol{\Theta}\right)$ is the conditional density at time $t$. 